\title{
STRUCTURAL HETEROGENEITIES IN GLASSY AND MOLTEN POLYMERS
}

\author{
H. G. ZACHMANN \\ Institut für Physikalische Chemie der Universität Mainz, \\ Mainz, GFR
}

\begin{abstract}
In molten polymers, three types of structural heterogeneities have to be considered: (1) heterogeneities caused by short range order; (2) fluctuating bundles of more or less parallel chains which are much larger than the domains with short range order; (3) small crystals which are thermodynamically not stable caused by heterogeneous fluctuations as discussed by Frenkel. The definition of the three types of heterogeneities, their mutual relation and the theories by which they are predicted are discussed. Special attention is given to the problem of characterization of bundles and to the question of the existence of bundles. Experimental methods, by which heterogeneities can be detected, are critically discussed and the results are reviewed.

In the glassy state, the heterogeneities mentioned above also occur. Here, however, they do not fluctuate but are frozen in. In some substances, in addition, ordered regions of another kind occur which disappear after heating above the glass transition temperature. The possible nature of these regions is discussed.
\end{abstract}

\section{A. INTRODUCTION}

The structure of amorphous polymers influences the mechanical behaviour as well as the crystallization process. Therefore, the investigation of that structure is of great interest.

Various types of structural heterogeneities in molten and glassy polymers have been discussed up to now. They are summarized in Table 1. First, we

Table 1. Types of order in molten and glassy polymers

Short-range order

Bundles of chains

Small unstable crystals ('heterogeneous fluctuations')

Special structures occurring only in the glassy state

mention the short-range order, which is already well-known from low molecular weight substances. Short-range order means that, within small regions, more or less regular distances between atoms occur. Secondly, one considers regions in which the chains have a preferential orientation without showing any crystalline order. Such structures are called chain-bundles. Thirdly, according to Frenkel, above the melting point thermodynamically unstable, 
fluctuating crystals are always formed. All these heterogeneities exist in the melt as well as in the glassy state. In the melt, due to thermal motion, the structures disappear and are formed again very quickly. In the glassy state they are frozen in.

In the glassy state, in addition to the structures frozen in from the melt some other structures can be formed by annealing below the glass transition temperature. They can be detected by mechanical measurement, calorimetry and electron microscopy and they disappear after the sample has been heated slightly above the glass transition temperature.

In what follows we will describe and classify the various heterogeneities and their mutual relations and we will discuss the theoretical and experimental evidence.

\section{B. SHORT-RANGE ORDER}

It is well known that in liquids, melts and glasses the molecules are not arranged completely randomly as in gases; there exists a so-called shortrange order ${ }^{1}$. We explain this effect first for the simple case of a liquid consisting of small spherical molecules. Figure 1(a) shows schematically the



(a)



(b)

Figure 1. Illustration of short-range order: (a) arrangement of atoms; (b) pair distribution function for liquid argon ${ }^{1,2}$.

arrangement of the molecules in the liquid. Due to the fact that the liquid has nearly the same density as the crystal, the volume in the liquid is almost completely filled with matter. Therefore, most of the molecules are in contact with their neighbours. This affects the distance distribution function between the molecules. Nearest neighbours have always almost the same distance. For second-nearest neighbours, third-nearest neighbours etc. the distance distribution function becomes gradually broader. As an example, Figure 1 shows the pair distribution function $\mathrm{g}(r)$ obtained by $\mathrm{x}$-ray scattering for liquid argon. $\mathrm{g}(r) \Delta v$ gives the probability of finding the centre of a molecule in a volume element $\Delta v$ in a distance $r$ of a molecule considered. One sees that all distances to the nearest neighbours lie within a very narrow range about $3.8 \AA$, the distances to the second nearest neighbours in a broader range and 
so on. Due to these regular distances a considerable amount of order is established within small regions.

For larger molecules, especially for polymers, the situation is more complicated. As one obtains from scattering experiments the distances of atoms, one has to distinguish between intramolecular and intermolecular distances. The intramolecular distances are in many cases quite regular because they are determined by the valence-bond-lengths and valence-bondangles. But also the intermolecular distances show some regularity which again has to be interpreted as short-range order due to space filling. Figure 2

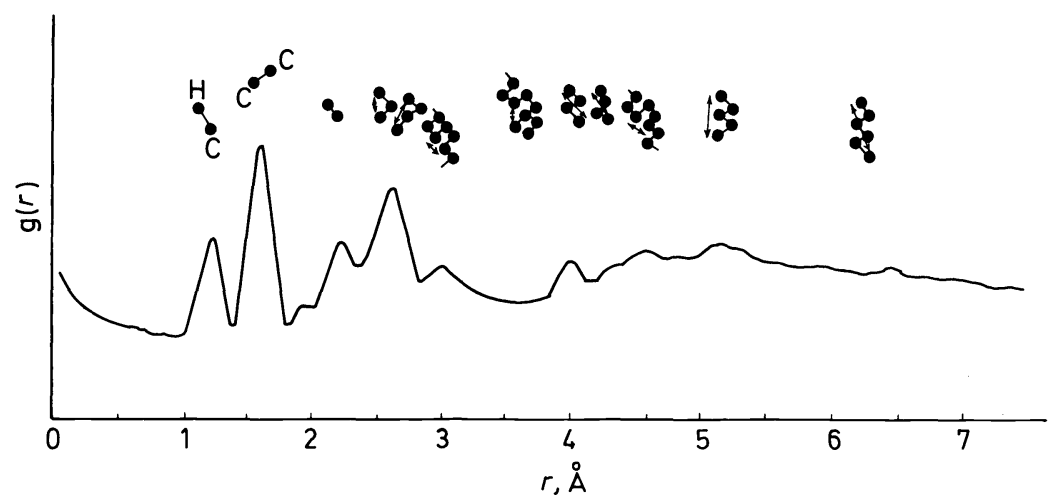

Figure 2. Pair distribution function for molten polyethylene ${ }^{3}$.

shows the pair distribution function for liquid polyethylene obtained by electron diffraction. One finds many peaks which are caused mostly by intramolecular distances as for example that at $1.2 \AA$, attributed to the $\mathrm{C}-\mathrm{H}$ distance. But there is also a broader reflection in the region of 4-6 $\AA$, which is caused by intermolecular distances. This peak corresponds to the 'amorphous halo' found with $\mathrm{x}$-ray scattering.

This example shows that short-range order exists also in polymers although its investigation is more difficult there.

\section{CHAIN-BUNDLES}

\section{(1) Definition and characterization of chain-bundles}

Many authors assume that the molecules in amorphous polymers are randomly coiled ${ }^{4-11}$, in the same manner as in a dilute solution. This is shown schematically on the left side of Figure 3 . On the other hand, other authors ${ }^{12-19}$ have come to the conclusion that bundles of chains with preferential orientation occur, as shown on the right side of Figure 3. A special model showing bundles with chain folding was proposed by $\mathrm{Yeh}^{15}$ and is shown in Figure 4 . A still more detailed model with bundles which are bent to form so-called 'meanders' was proposed by Pechhold and Blasenbrey ${ }^{19}$. At the present time much controversy is going on concerning the question whether bundles exist or not. Before discussing the theoretical and experimental evidence 


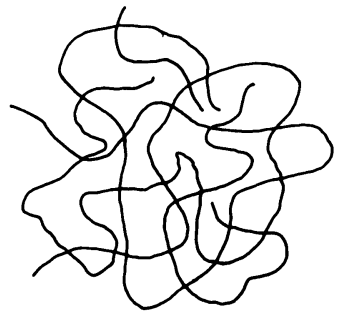

(a)

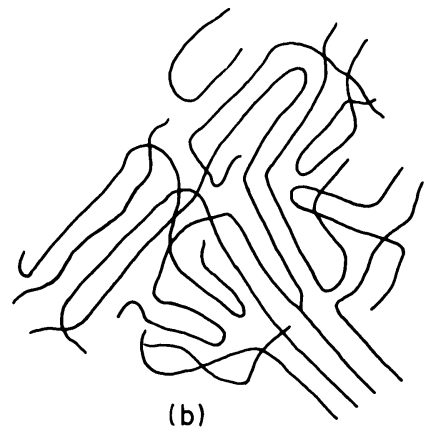

(b)

Figure 3. Schematic representation of different possibilities for the arrangement of chains in amorphous polymers: (a) randomly coiled chains; (b) bundle model.

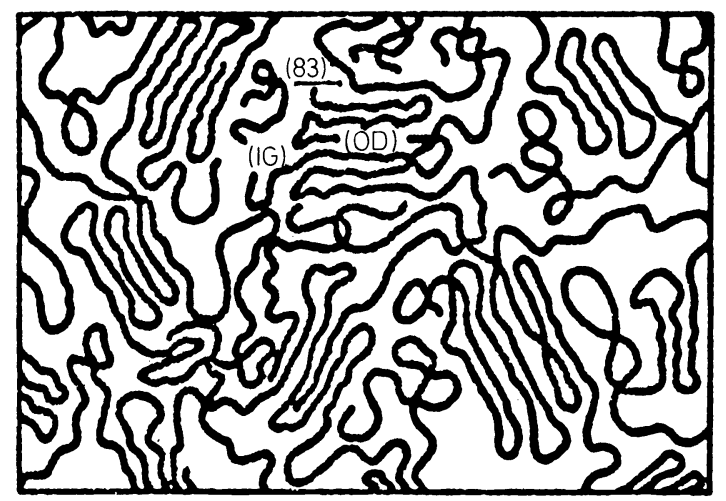

Figure 4. Bundles with folded chains in amorphous polymers according to $\mathrm{Yeh}^{15}$.

for bundles and randomly coiled molecules we have to treat the problem how to define and characterize a bundle.

It is important to state that bundles are not crystals. Though the molecules show a preferential orientation, there does not exist any long-range order. As an example Figure 5(a) shows a non-crystalline bundle of chains in a two-dimensional cubic lattice. In an ideal crystal all the bonds would lie in the $z$-direction, and the distance between two chains would be everywhere the same [see Figure 5(d)]. Here in the bundle a considerable amount of bonds is lying in the $x$-direction and different distances occur. The bundle character follows simply from the fact that each step in the $\oplus x$-direction is compensated after one or two steps in the $z$-direction by a step in the $\ominus x$ direction. In principle it is possible that the number of bonds in the $\oplus x$ and - directions is the same as that in the $z$-direction so that the bundle does not show any anisotropy.

In addition, one must also mention that bundles are not identical with short-range order regions. The short-range order in the chain-direction in the bundle is not better than that in any other direction. Randomly coiled 


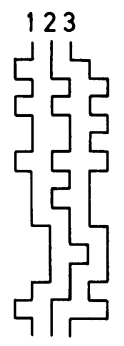

(a)



(b)

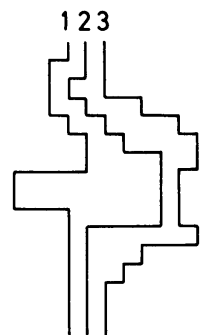

(c)
123

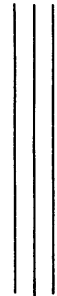

(d)

Figure 5. (a), (b), (c): Three arrangements of cubic lattice chains with equal numbers of bonds in $+x,-x$ and $z$ directions but different bundle characters; (d): arrangement of chains in the ideal crystal.

chains can have the same degree of short-range order as chains within a bundle. This is so because, within every small region of short-range order, there is no difference between randomly coiled chains and chains within a bundle with respect to arrangement of atoms. A difference between the two models is noticed only if one follows the chains for much longer distances.

To characterize a bundle one must find a measure for the amount of parallelism or, as we can say, the bundle character. One has first to find the ideal direction for the chains. In the example of Figure 5(a) this is the $z$ direction. Next, one has to measure the deviations from this direction. This cannot be done simply by the fraction of steps in directions other than the ideal one. This is seen clearly if one compares the three arrangements (a), (b) and (c) in Figure 5. Chain 1 has the same number of steps in each direction in bundle (a), bundle (b), and bundle (c). The same is true for chain 2 and chain 3. In spite of that, in (a) we have more bundle character than in (b) and here more than in (c). Not only the number of steps in different directions influences the bundle character, but also how the different steps follow after each other. A better measure for the bundle character is perhaps the second moment of the distribution of the distances between neighbouring bonds in the $z$-direction.

In addition to the amount of parallelism we have to know the dimensions of the bundle. One has to distinguish between the lateral dimension, given by the number of chains, and the longitudinal dimension given by the number of units of each chain within the bundle in the bundle direction. One sees from the simple examples shown in Figure 5 that the dimensions will depend on how bundles are defined. If only a little parallelism of the chains is demanded we may say that (b) is one big bundle. If we demand good parallelism we may see two smaller bundles in (b).

Another quantity characterizing the bundle is the amount of chain folding on the surface.

Very important also is the average lifetime of an individual bundle. Far below the glass transition temperature, in principle, each bundle will be stable for long periods of time. In the melt, however, due to changes in the conformations of the chains, the bundles will constantly disappear and will 


\section{H. G. ZACHMANN}

be formed again with other preferential orientations and at other places. We say that the bundles fluctuate. The average lifetime of a bundle is an important quantity. For many properties as for example viscosity, crystallization and mechanical behaviour it makes an essential difference whether the lifetime is for example three minutes or $10^{-6}$ second.

Table 2. Quantities of the characterization of chain-bundles

Amount of parallelism of the chains

Lateral dimensions (number of chains)

Longitudinal dimensions (number of units in each chain in bundle direction)

Amount of chain folding

Average lifetime

(2) Theoretical and experimental evidence for randomly coiled chains and for bundles

(a) Space filling problems

The density of a liquid is only about ten per cent less than that of a crystal. This comparatively high value has important consequences for the structure and the question of order.

A first consequence, the existence of short-range order, has been already discussed. Another important effect arises for small rod-like molecules. This was shown first by Rehaag and Stuart ${ }^{20}$ many years ago. Models of such molecules were shaken and the arrangement of the molecules obtained was investigated as a function of density. Figure 6 shows the results. For small densities, the orientations of the rods were distributed randomly [see Figure

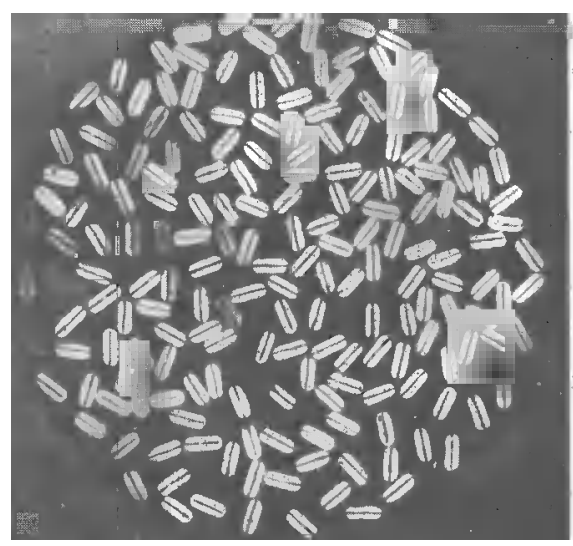

(a)

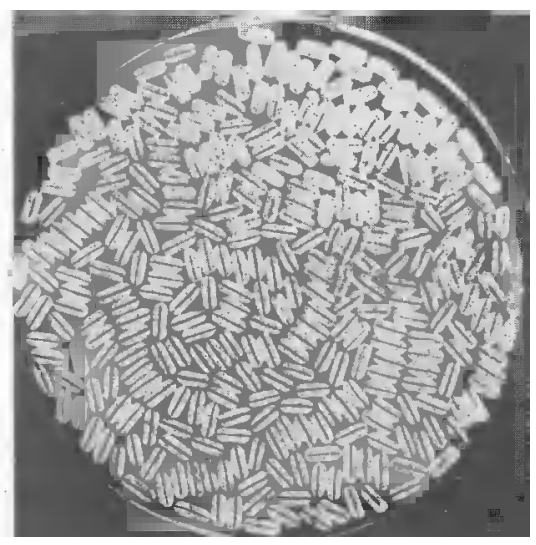

(b)

Figure 6. Arrangements of rods(a) in a system of low density and (b) in a system of high density ${ }^{20}$. 
6(a)]. For high concentrations, regions of parallel rods are formed [see Figure 6(b)]. The tendency for the formation of such regions increases if attractive forces are built into the molecules by using small magnets ${ }^{21}$.

In recent times we have calculated the size distribution of such ordered regions by using a lattice model described elsewhere ${ }^{22,23}$. Figure 7 shows the



Figure 7. Fraction of rods lying in ordered regions consisting of $n$ parallel rods as a function of $n$. $\gamma$ is the intermolecular energy measured in $\mathrm{kT}$.

fraction of molecules $q_{n}$ lying in ordered regions of $n$ parallel molecules as a function of $n$. The parameter $\gamma$ is the intermolecular energy in $k T$ units. If $\gamma$ is zero, one sees that $q_{n}$ decreases with increasing $n$. For example, approximately 15 per cent of the molecules lie in ordered regions consisting of three molecules and about one per cent in regions consisting of six molecules. The crosses indicate 'experimental' results, which are obtained from Figure 6(b). With increasing intermolecular attraction $\gamma$ the number of larger ordered regions increases.

For polymer melts the consequences of the high density were first investigated by Robertson ${ }^{16}$. This author started with a consideration of rods of length $l$ and cross section diameter $d$ (see Figure 8 ). The angle between two rods is called $\theta$. The larger $\theta$ the more space must be attributed to one rod. Robertson derived a relation between the largest possible angle $\theta$ and the density of the system. Figure 8 shows the results. Plotted is this angle $\theta^{*}$ as a function of the relative density, that is the density of the liquid $d_{\mathrm{a}}$ divided by the density of an ideal crystal $d_{\mathrm{c}}$. The results depend on the ratio of the length of the rods to the diameter. For example, for $l / d=1$ and a relative density of 0.850 the value of $\theta^{*}$ is about $14^{\circ}$. This corresponds to almost parallel alignment.

To apply these results to chain molecules, Robertson assumed that chain 

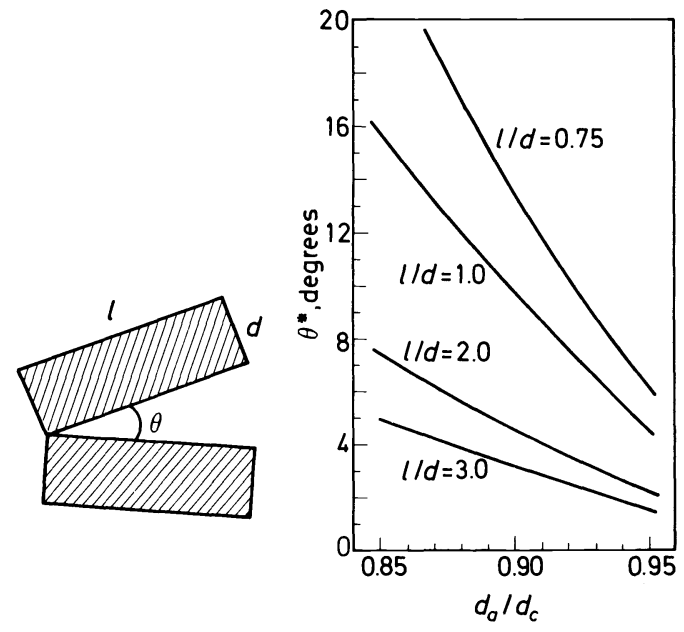

Figure 8. Maximum angle $\theta^{*}$ for several values of $l / d$ as a function of the ratio of amorphous to crystalline densities ${ }^{16}$.

molecules have to be divided into such segments that the ratio $l / d$ is approximately unity. From this he deduced also that chain molecules must lie almost parallel and must form bundles.

Fischer ${ }^{24}$ objected to the calculations of Robertson that some error was made when the volume necessary for a pair of rods was averaged over the angle $\theta$. Using the right averaging, one obtains for $l / d=1$ a density which is close to the measured value, without assuming any parallelism. Parallelism of the rods has to occur only for larger values of $l / d$. From this it follows that, if one assumes $l / d=1$, bundles do not have to be formed. The assumption of Robertson that, in polymers, $l / d$ is approximately one is somewhat questionable, however.

There is in addition another objection to the conclusions of Robertson, pertinent for all values of $l / d$. From the way the calculations were performed, it follows that, in the polymer, any parallelism obtained concerns only the small segments of length $l$. This is seen most clearly when one considers again the arrangement of rods in Figure 6(b). According to Robertson the rods in each ordered region must have within some limits depending on $l / d$ the same direction. But not all the rods of the sample must have this direction. The different regions may be arranged more or less randomly. In the same way, it follows for polymers that short parts of the chain of length $l$ have to lie parallel rather than longer parts. No result is obtained for the arrangement of larger parts of the chain. Therefore, the question whether bundles have to be formed simply because of lack of space is not answered by the investigations of Robertson.

One could try to find an answer to this question by packing wooden models of molecules into a box. But one needs for this a tremendous number of molecules. Therefore we decided to perform such a filling experiment by computer simulation ${ }^{25}$. A primitive cubic lattice of 10000 points was assumed. 
Each molecule consisted of 100 beads connected with each other. The computer introduced bead after bead into the lattice, so that each bead was lying on one lattice point. For the first bead of each molecule the computer chooses at random any free point in the lattice. For the following beads, the computer had to choose one of the five lattice points which are the next neighbours to the lattice point occupied by the bead introduced previously. Every time a place chosen was occupied, the computer tried again. If all five places turned out to be occupied the computer shifted some beads lying in the vicinity of empty points. By this procedure it was possible to occupy 87 per cent of the lattice points. This corresponds to the density usually found in liquids.

Figure 9 shows the average square end-to-end distance of the chains introduced into the lattice as a function of the volume fraction of the chains introduced during filling, that is the fraction of occupied lattice points.

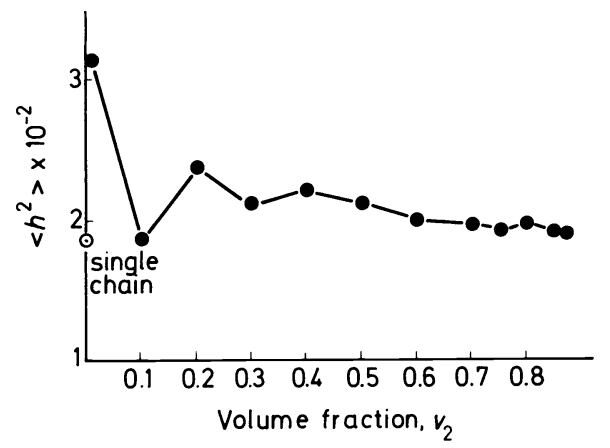

Figure 9. Average square end-to-end distance as a function of the volume fraction $v_{2}$ of the chains introduced into the lattice ${ }^{25}$.

The open point on the ordinate gives the value for a single chain in the lattice, averaged over 298 filling procedures. One sees that the values for high concentrations, corresponding to the melt, are almost the same as for the isolated, single chain. Some deviations occur for smaller concentrations; these are due, however, to statistical fluctuations occurring because the number of chains for which the average is calculated is smaller. From this we conclude: In the melt, arrangements of the chains are possible in which the chains have the same average square end-to-end distance as in a dilute solution.

In order to obtain more detailed information, we wanted to know if some additional stretching of the chains could occur when the concentration increases. Therefore we determined the straight parts of the chains of different lengths. Figure 10 shows the fraction of bonds lying in straight parts of lengths $\zeta$ as a function of the fraction of lattice points occupied during filling. One sees that about 60 per cent of the bonds are lying in straight parts of length 1,26 per cent in straight parts of length 2 and only a few per cent in the longer straight parts. These percentages do not depend on the concentration. Therefore no stretching of the chains is necessary in order to increase the concentration of chains to the value found in the melt.

In addition, the formation of bundles with completely parallel chains was 


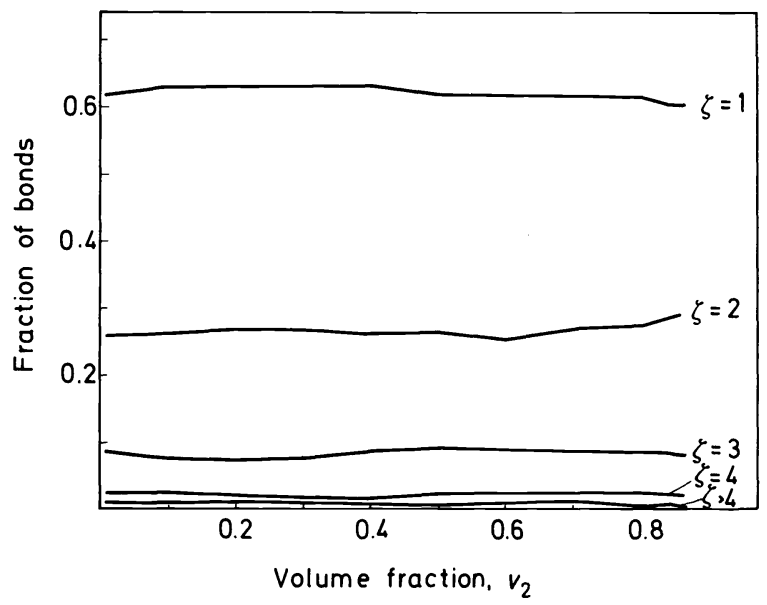

Figure 10. Fraction of bonds lying in straight parts of length $\zeta$ of the chains as a function of the volume fraction $v_{2}$ of the chains introduced into the lattice ${ }^{25}$.

investigated. Such bundles are characterized by two values: the number $n$ of chains forming the bundle as a measure for the lateral dimensions and the number $\zeta$ of bonds in the $z$-direction in each chain as a measure for the height of the bundle. Figure 11 shows the fraction of bonds lying in bundles as a function of $n$. Let us consider first the bundles of height $\zeta=2$. For example, 3 per cent of the bonds lie in bundles consisting of three chains, 0.4 per cent in bundles consisting of five chains and a negligible amount in larger bundles.

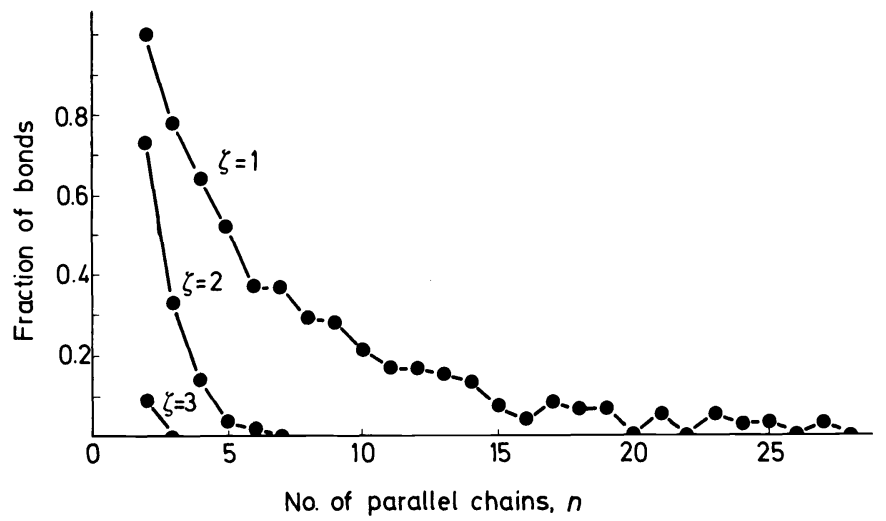

Figure 11. Fraction of bonds lying in bundles as a function of the number $n$ of parallel chains forming the bundle. $\zeta$ is height of the bundle in number of bonds ${ }^{25}$.

Bundles of height $\zeta=3$ are still less frequent. The majority of the bonds lie in 'bundles' of height 1 , that means in 'bundles' with a single bond in the bundle direction. Such small regions cannot be considered to be the bundles usually discussed. Therefore we conclude: Even in the melt, it is possible to 
arrange the chains in such a way that they are randomly coiled and no bundles are formed. The argument that bundles have to be formed because of lack of space is not valid.

At the present time we are also investigating the formation of bundles with defects. We also want to study the situation arising in a diamond lattice. But we think that these investigations will not change the above result in essence.

One is not allowed to conclude from this result, however, that bundles do not occur. First no attractive forces have been considered which may favour bundle formation. Secondly, the calculated conformations are not necessarily those corresponding to thermodynamic equilibrium. Due to the fact that, during filling, further attempts were made and even chains had to be moved after an unsuccessful step. our calculation was not a Monte Carlo determination of the equilibrium state. It serves only to prove that arrangements with random conformations do exist in dense systems.

Some other space problems occurring with dense systems including the impingement of bundles were investigated by Anthony and Kröner ${ }^{26}$. If bundles impinge on each other different situations may occur with respect to the continuation of the chains. They may just bend, as indicated in Figure 12(a), or loops may occur as seen in Figure 12(b). Using a continuum

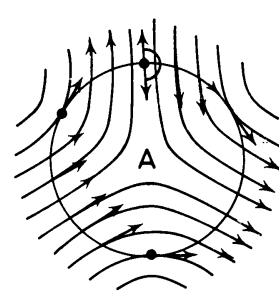

(a)



(b)

Figure 12. Different situations in the impingement of bundles ${ }^{26}$.

theory, Anthony and Kröner classified and investigated the different possibilities of bundle arrangements. One must stress, however, that their theory does not prove that bundles exist. The existence of bundles is assumed and the consequences are investigated.

(b) Melting, rubber elasticity and viscosity

The melting of polymers as a transition from the crystalline state to a melt with randomly coiled chains has been investigated by different authors 22,27 . It was possible to show that a first order transition can be expected in this case. This result remains true if one assumes that small, fluctuating bundles occur in the melt. Problems arise, however, if one investigates the transition from a crystal to a bundle which does not fluctuate with the same dimensions as the crystal. In this case, the only difference between the crystal and the melt is the number of defects (see Figure 13). 


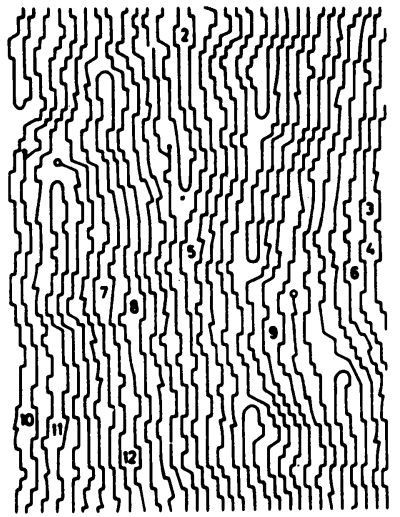

(a)

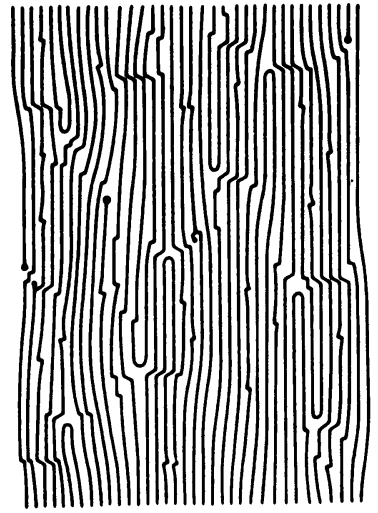

(b)

Figure 13. Arrangement of chains in the melt (a) and in the crystal (b) according to Pechhold and Blasenbrey ${ }^{18,28}$.

Pechhold and Blasenbrey ${ }^{28}$ proved that there can take place a first order transition between a crystal with a low concentration of defects and a 'crystal' with a large concentration of defects, which can be considered as a melt. Bauer ${ }^{29}$, however, remarked that the assumptions made in this calculation are not very realistic.

Of interest also is the influence of bundles in rubber elasticity. As is well known, rubber elasticity can be explained quite well by the randomly coiled chain model of the melt. In addition it turned out that the assumption of small fluctuating bundles could improve the agreement between theory and experiment ${ }^{30}$. It seems, however, difficult to explain the big entropy change observed with the stretching of the sample if big, stable bundles are assumed, especially as considered in the meander-model.

Again, stable bundles do not explain the dependence of the melt-viscosity on the molecular weight ${ }^{5}$.

In summary, we see that melting, rubber elasticity and viscosity can be explained by randomly coiled chains and, if one wishes, also by superimposed small fluctuating bundles. Difficulties arise, however, if one assumes big, stable bundles.

\section{(c) Electron microscopy}

Some indication for the existence of bundles is obtained from observations with the electron microscope. Different authors have reported that in amorphous polymers small balls can be observed ${ }^{12,14,31}$. Figure 14 shows such a ball-like structure observed by Yeh and Geil ${ }^{14}$ on a surface replica of amorphous polyethyleneterephthalate. The balls have on average a diameter of $75 \AA$.

According to Yeh and Geil ${ }^{14}$ the balls can be observed also in transmission electron microscopy on thin films. Of special interest is the fact that one can see also some of the balls in the dark field by using a part of the innermost diffuse scattering ring. This shows that the chains in the balls have a preferential orientation. 


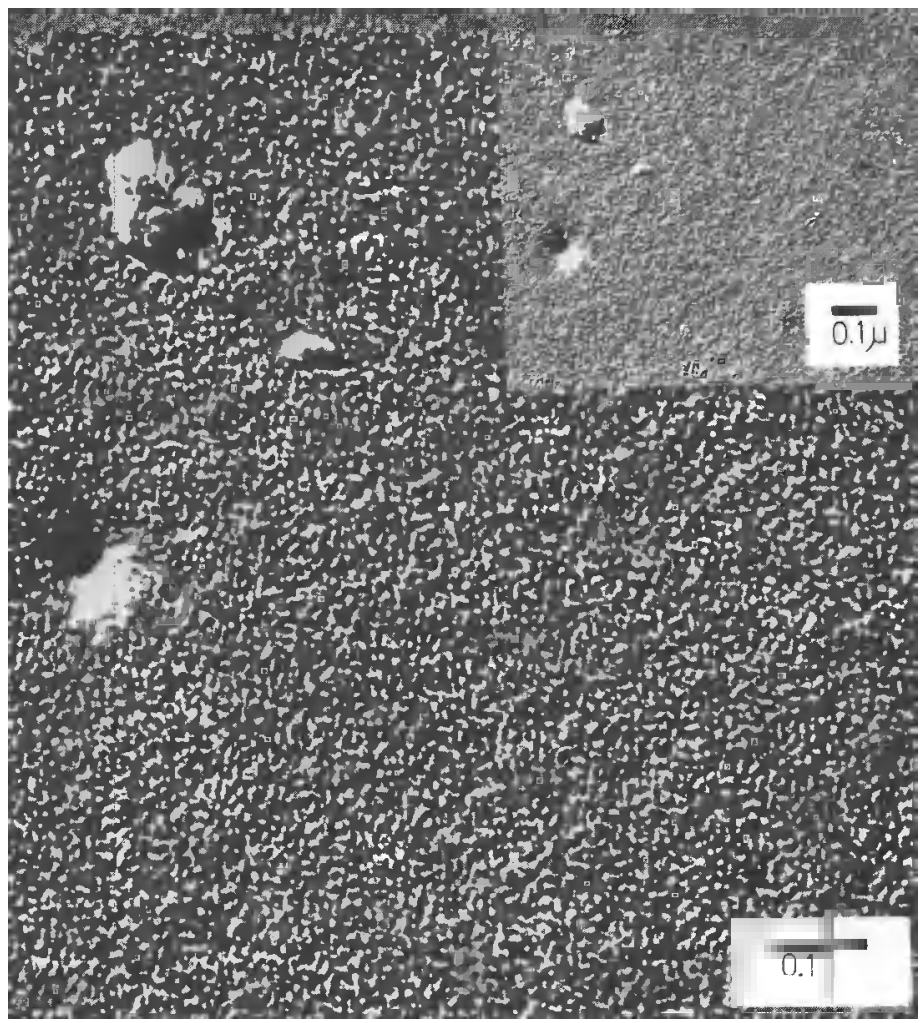

Figure 14. Surface replica of amorphous polyethyleneterephthalate showing the presence of ball-like structures ${ }^{14}$.

Quite recently, Yeh ${ }^{15}$ reported the observation of balls in the dark field in amorphous polymers above the glass transition temperature. This means that bundles with preferential orientation are stable for a longer time even in the melt. It seems difficult to bring this result into agreement with the short lifetime of a bundle deduced from n.m.r. experiments (see next section).

\section{(d) Nuclear magnetic resonance}

The shape and the halfwidth of the n.m.r. line depend strongly on the motion of the molecule. Rigid molecules give a broad line. The broadening is caused mainly by the local magnetic fields of the protons. If by the motion of the molecules these local fields are averaged to zero within the lifetime of a spin, the line becomes very narrow.

In the melt of polyethylene, one finds a very narrow line with a halfwidth of about $20 \mathrm{mG}$. This shows that the local fields are averaged to zero' ${ }^{17}$. What conclusion can be drawn from this result with respect to bundle structure?

Let us consider a $\mathrm{CH}_{2}$-group in polyethylene. In order that the magnetic field of the proton 1 acting on the proton 2 be averaged to zero the distance 


\section{H. G. ZACHMANN}

vector $\mathbf{r}$ between the two protons (see Figure 15) has to show all possible orientations, within the transition time of a spin that is approximately $10^{-4}$ second. For chains which change their conformations quickly this condition is fulfilled. It is obvious, however, that for chains within a bundle such a motion is not possible. This has been proved by us also by exact calculations ${ }^{32,33}$.

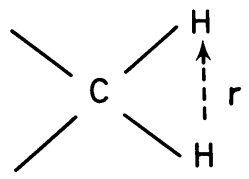

Figure 15. Definition of the vector $\mathbf{r}$ in the $\mathrm{CH}_{2}$-group.

We have determined the second moment of the n.m.r. line of chains within a bundle by taking into account all the possible changes of chain conformations and motions of the $\mathrm{CH}_{2}$-groups. We obtained as a result (see Table 3) that even for bundles which show large distortion the second moment cannot be smaller than about $1.3 \mathrm{G}$. This corresponds in the case of a Gaussian line to a linewidth of $1.1 \mathrm{G}$. This is much more than is found experimentally.

Table 3. Bundle model and width of n.m.r. line

Linewidth of polyethylene melt (experimental):

$20 \mathrm{mG}$

Calculated second moment of the line for a stable bundle:

$>1.3 \mathrm{G}^{2}$

This corresponds in the case of a Gaussian line to a linewidth of:

$>1.1 \mathrm{G}$

On the other hand, if we assume that the bundles fluctuate so quickly that every part of a chain lies within the lifetime of a spin within many bundles with different orientations, the line may become as narrow as is observed experimentally. So we have to conclude the following: If bundles exist, their average lifetime must be smaller than the transition time of a spin, that is approximately $10^{-4}$ second.

Nuclear magnetic resonance also provides some evidence that fluctuating bundles really do exist. This can be concluded from studies of the shape of the line ${ }^{17,33}$. From the n.m.r. theory one knows the following (see Table 4): If the motion of all $\mathrm{CH}_{2}$-groups can be described by a single correlation time one obtains a Lorentzian line. The halfwidth of the line decreases with increasing correlation time. If, however, two types of $\mathrm{CH}_{2}$-groups with different correlation times are present one obtains a superposition of two Lorentzian curves with different halfwidths.

Now, in a dilute solution of polyethylene we find experimentally a Lorentzian line [see Figure 16(a)]. In concentrated solutions and in the melt, however [see Figure 16(b)], the line obtained can be separated into two Lorentzian curves with different halfwidths ${ }^{17,33}$. We can explain the superposition of two Lorentzian lines by the formation of bundles: The $\mathrm{CH}_{2}$ groups within the bundles and those of tie molecules have a comparatively large correlation time of motion because the correlation time is determined 

One correlation time $\tau$
for all $\mathrm{CH}_{2}$-groups
$\quad \delta H \sim \tau$

Two kinds of $\mathrm{CH}_{2}$-groups with different correlation times $\tau_{1}, \tau_{2}$ superposition of two Lorentzian lines
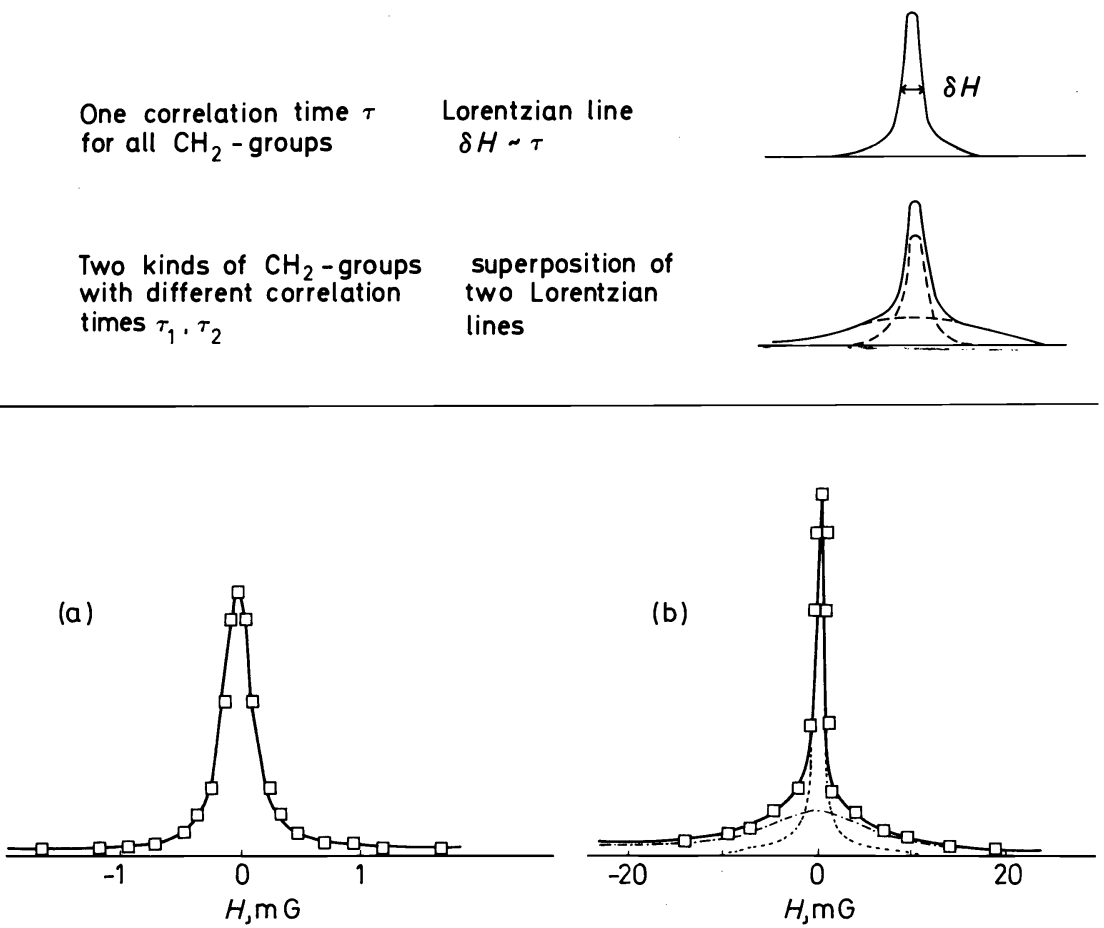

Figure 16. High resolution n.m.r. line of (a) polyethylene in solution and (b) molten polyethylene ${ }^{17}$. The solid lines give the experimental results. The points represent in (a) the fitted Lorentzian line and in (b) the sum of the two Lorentzian lines (dashed and chain-dotted) which lead to a best fit.

by the lifetime of the bundles. Therefore these groups give rise to a comparatively broad Lorentzian line. The $\mathrm{CH}_{2}$-groups lying in highly mobile parts of the chains outside the bundles have a small correlation time and give rise to a narrow Lorentzian line. This interpretation is supported also by the following result ${ }^{17}$ : We observed that during isothermal crystallization first the broad component decreases, which is interpreted as a crystallization of the bundles. Later, very slowly, the narrow component decreases, which is interpreted as a crystallization of highly mobile chains outside the bundle.

Investigations with higher resolution have shown that the line of the melt consists of more than two Lorentzian components. This does not change the main conclusions, however. Therefore, n.m.r. provides some indication of the existence of bundles although, of course, bundles are not the only possible explanation for the observed results.

\section{(e) Electron diffraction}

In 1969 Kargin and co-workers ${ }^{13}$ published the radial distribution function of molten polyethylene obtained by electron diffraction. This distribution showed several sharp peaks in the region from 4-6 $\AA$ which obviously 
were caused by regular intermolecular distances. This result was seen as a strong evidence for the existence of bundles with regular distances between the chains.

Quite recently, however, Voigt-Martin and Mijhoff ${ }^{3}$ reported that the above-mentioned peaks in the area between 4 and $6 \AA$ are spurious effects caused by integration limits in the Fourier transform. A correct Fourier transform gives only a broad peak (see Figure 2) as must be expected from normal short-range order. According to this result electron diffraction measurements can no longer be seen as a proof of bundles with regular distances between the chains. On the contrary, they prove that such bundles do not exist. On the other hand, loose bundles as considered in Figure 5(a) with no regular long-range distances can exist. With electron diffraction such bundles cannot be detected because they influence neither the long-range nor the short-range order.

\section{(f) Light scattering}

Light scattering can be caused by density fluctuation and by anisotropy.

Let us discuss first the anisotropy scattering. A bundle of ideal parallel chains should have a considerable amount of anisotropy and therefore cause a much more intensive light scattering than randomly coiled chains. If parallelism is not so good, the light scattering should decrease. Dettenmeyer and Fischer ${ }^{9}$ have shown that the anisotropy light scattering of amorphous polymethylmethacrylate is rather small. Therefore, if chain bundles exist in this substance, the anisotropy of the bundle must be very small. The same conclusion has to be drawn also from measurements of the birefringence in a magnetic field ${ }^{11}$.

It is not difficult to construct bundles with very low anisotropy in the case of the cubic lattice chain shown in Figure 5. The construction of almost anisotropic bundles of chains in a diamond lattice seems to be more difficult.

The investigated substance polymethylmethacrylate does not crystallize. Of special interest is the amount of anisotropy light scattering of crystallizable substances in the molten or glassy state. Measurements on such samples have not yet been performed.

The light scattering of amorphous polymethylmethacrylate caused by density fluctuations ${ }^{9}$ is also small. This shows that bundles, if they exist, must have almost the same density as their surroundings. This condition can be fulfilled in any chain model without difficulties.

\section{(g) Neutron scattering}

Interesting information on the conformations of the molecules in the bulk material can be obtained by neutron scattering. In order to exclude intermolecular scattering, one has to use a solution of a normal polymer in a deuterated polymer. One can then determine the dimensions of the chain in the bulk material in the same way as in the dilute solution.

Scattering experiments have been performed so far on polymethylmethacrylate $^{6,7}$ and on molten polyethylene ${ }^{8}$. In both cases it was found that the radius of gyration in the bulk material is approximately the same as in the dilute solution. In addition, from the angle-dependence of the scattered intensity, it was concluded that gaussian statistics applies to the conformations. 
The formation of longer, straight parts of the chains in bundles would increase the statistical segment and thus also the average square end-to-end distance and the radius of gyration ${ }^{4,34}$. Furthermore, such straight parts of chain would also cause deviations from gaussian statistics. Therefore, neutron scattering seems to prove that the chains are randomly coiled. This conclusion, however, is not compelling. Yeh ${ }^{15}$ has pointed out that the effect of increasing the end-to-end distance could be compensated by the introduction of folds into the chains. Concerning gaussian statistics, one has to investigate how strictly this has to be followed in order to obtain the observed angle-dependence of the scattered intensity. It might be possible, perhaps, to bring these statistics into agreement also with the bundle structure theory.

\section{(h) Conclusions}

To summarize we can say the following (see Table 5): Dense packing of the chains does not imply as a necessary consequence the formation of bundles.

Table 5. Present results concerning the bundle problem

Requirement for space filling: No bundles necessary, but existence of bundles possible.

Melting, rubber elasticity, viscosity: Explanation seems difficult with big stable bundles.

Electron microscopy (on PET, PC etc.): Small balls, visible also in the dark field, indicate bundles.

Nuclear magnetic resonance (on PE): Indications of bundles. Bundles have to fluctuate if they exist.

Electron diffraction (on PE): If bundles exist, they show no sharp intermolecular distances.

Light scattering (on PMMA): If bundles exist, they must have a very small anisotropy,

Neutron scattering (on PMMA, PE): If bundles exist, the radius of gyration of a chain must be the same as for randomly coiled chains.

Bundles may be formed but it is also possible to pack the chains in completely random conformations. Electron microscopy añd n.m.r. experiments give some more or less strong evidence for the existence of bundles. But if they exist there are important restrictions concerning their properties. They have to fluctuate. No crystal-like intermolecular regular distances occur. The anisotropy must be very small. The average end-to-end distance of the chains must be approximately the same as in randomly coiled chains. Of course these results can be applied strictly only to the substances investigated in each case. It has been shown that it might be possible to fulfil all these conditions.

\section{SMALL, UNSTABLE CRYSTALS}

Besides short-range order and bundles one has to consider a third effect: the 'heterogeneous fluctuations' discussed first by Frenkel ${ }^{35}$ many years ago. Owing to thermal fluctuations above the melting point, small crystals are created and disappear again very quickly. No individual crystal is stable itself, but there exists a constant distribution of crystals of different sizes. Such 
crystals are called crystal embryos or cybotactic structures. The size distribution of crystal embryos can be calculated by minimizing the free energy of the system.

Some scientists do not accept at all the existence of different phases in the melt. They doubt that thermodynamics can be applied to such small systems as crystal embryos. The majority of scientists, however, use the concept of Frenkel successfully to explain crystallization kinetics.

I will not go into further detail because the basic concept of Frenkel is well known and often discussed. I want to point out only the following: The crystal embryos are neither identical with short-range order nor with chainbundles. The order in a crystal embryo extends over much larger distances than short-range order. It is much better developed than the order within a bundle. In spite of these differences, however, the process of crystal embryo formation is strongly influenced by the conformation of the chains in the polymer. If bundles are present, it seems reasonable to assume that the crystal embryos grow out of the bundles ${ }^{36}$. The transformation of a bundle to a crystal is connected with much less diffusion than the formation of a crystal out of randomly coiled chains. Therefore the existence of bundles should greatly enhance the crystallization process. The amount of folds in the bundles should influence the morphology of the crystalline material obtained $^{37}$.

\section{E. SPECIAL STRUCTURES OCCURRING ONLY IN THE GLASSY STATE}

As already pointed out, we find in the glassy state short-range order, small crystals and, perhaps, bundles. In contrast to melt conditions all these structures do not fluctuate; each individual arrangement exists permanently.

Quite recently many experiments have shown that annealing below the glass transition temperature may result in additional structures. These structures are destroyed again if one heats up the sample for a short time above the glass transition temperature.

Let us consider first some results in electron microscopy. Yeh and Geil ${ }^{14}$ observed small balls in amorphous polyethyleneterephthalate. After annealing at temperatures below the glass transition temperature the balls formed bigger units. In amorphous polycarbonate Goddard, Frank and Stuart ${ }^{38,39}$ observed small balls which increased in size upon annealing below the glass transition temperature (see Figure 17). After heating up to $160^{\circ} \mathrm{C}$, the large balls disappeared and a fine-grained structure reappeared.

In agreement with these observations one finds in mechanical measurements that some secondary maximum in the loss modulus disappears and Young's modulus ${ }^{38,39}$ increases upon annealing below the glass transition temperature.

Further evidence for additional structures comes from calorimetric experiments. Hoffman and Knappe ${ }^{40,41}$ have measured the heat capacity of polyvinylchloride containing different amounts of dioctylphthalate (DOP) (see Figure 18). The full curves give the heat capacities for samples heated up immediately after slow cooling. These curves show a step in the glass transition temperature without any additional features. The dotted lines represent 


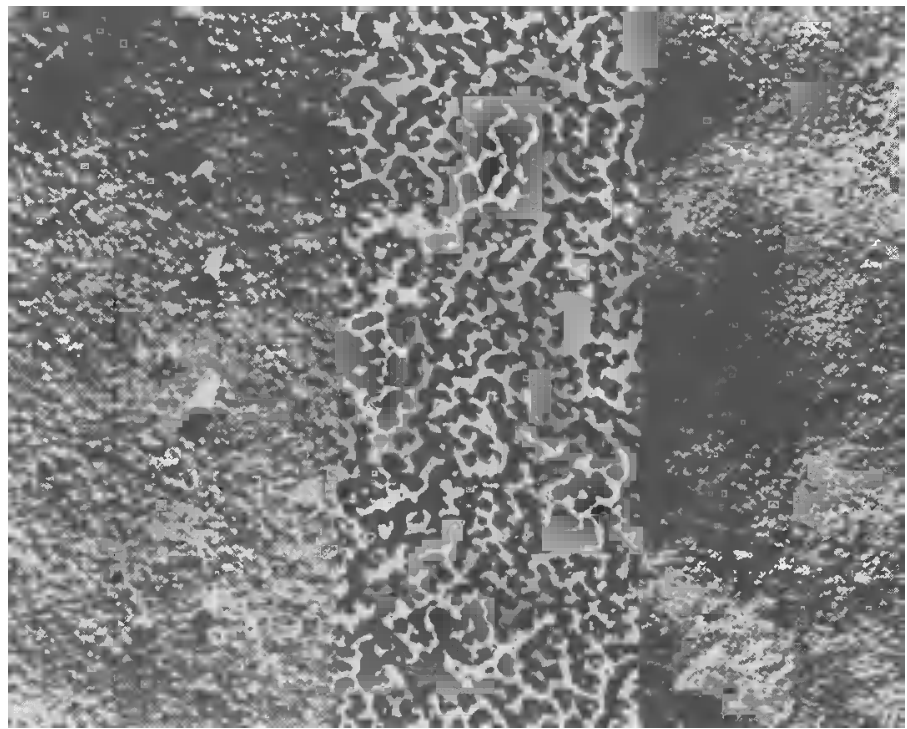

(a)

(b)

(c)

Figure 17. Structure in amorphous polycarbonate as revealed by electron microscopy ${ }^{35}$ : (a) untreated, (b) annealed 72 hours at $110^{\circ} \mathrm{C}$, (c) after additional annealing at $160^{\circ} \mathrm{C}$ for $20 \mathrm{~min}$ and quenching.



Figure 18. Heat capacity of polyvinylchloride with different amounts of DOP as a function of temperature ${ }^{40}$. Full line: heating after slow cooling; broken line: heating after slow cooling and annealing for five months at room temperature. 
the results for samples which were annealed for five months at room temperature before heating. A peak appears here in addition. According to Hoffmann and Knappe this peak is an indication that some order was formed during annealing below $T_{\mathrm{g}}$ and that an additional amount of heat is necessary in order to 'melt' the structures formed.

In the interpretation of peaks in the glass transition region one must be very careful. If the heating process occurs more quickly than the cooling process a peak may be caused by so-called enthalpy relaxation effects ${ }^{42,43}$. Hoffmann and Knappe showed, however, that this does not occur for the peaks mentioned. First: Enthalpy relaxation effects should disappear with decreasing heating rate. In their experiments the authors found that the area under the peaks decreased a little with decreasing heating rate, but even when one extrapolates to heating rate zero an appreciable peak remains. Secondly: The enthalpy relaxation peak should occur slightly above the end of the glass transition region. At least one of the peaks already occurs at lower temperatures. Therefore also the calorimetric measurements seem to prove that some additional order is formed upon annealing below the glass transition temperature.

What is the nature of this order? One is tempted to say that small crystals are formed. But the fact that these crystals disappear so far below the melting temperature shows that some precursor of crystals may be formed rather than real crystals. It is possible for example that groups with some interaction like phenyl groups are aligned together. Due to the small interaction energy these groups desintegrate immediately after segmental motion becomes possible.

From this it is seen that the field of structural heterogeneities in molten and glassy polymers still provides more questions than answers. Therefore, this field is a very interesting and important object of investigation.

\section{REFERENCES}

${ }^{1}$ F. Kohler, The Liquid State, Verlag Chemie: Weinheim (1972).

${ }^{2}$ D. G. Henshaw, Phys. Rev. 105, 976 (1957).

3 I. Voigt-Martin and F. C. Mijhoff, J. Appl. Phys. 46, 1165 (1975).

${ }^{4}$ P. J. Flory, IUPAC Symposium Macromolecular Chemistry-8, 1. Butterworths: London (1973).

5 M. Hoffmann, Makromol. Chem. 144, 309 (1971).

6 J. Schelten, W. A. Kruse and R. G. Kirste, Kolloid-Z. Z. Polymere, 251, 919 (1973).

7 R. G. Kirste, W. A. Kruse and K. Ibel, Polymer, 16, 12 (1975).

8 G. Lieser, E. W. Fischer and K. Ibel, Polymer Letter, 13, 39 (1975).

9 M. Dettenmaier and E. W. Fischer, Kolloid-Z. Z. Polymere, 251, 922 (1973).

10 J. H. Wendorff and E. W. Fischer, Kolloid-Z. Z. Polymere, 251, 876 and 884 (1973).

11 K. Dransfeld, Z. Z. Schickfuß, E. W. Fischer and J. H. Wendorff, to be published.

12 V. A. Kargin, A. I. Kitaigorodoskii and G. L. Slonimkii, Kolloid-Z. 19, 131 (1957).

13 Yu. K. Ovchinnikov, G. Markova and V. A. Kargin, Vysokomol. Soed. A11 (No. 2), 239 (1969).

14 G. S. Y. Yeh and P. H. Geil, J. Makromol. Sci., Phys. B1(2), 235 (1967).

15 G. S. Y. Yeh, J. Macromol. Sci., Phys. B6(3), 465 (1972).

16 R. E. Robertson, J. Phys. Chem. 69, 1575 (1965).

17 H. G. Zachmann and W. L. F. Gölz, J. Polym. Sci. C: Polymer Symposia No. 42, 693 (1973).

18 W. Pechold, Kolloid-Z. Z. Polymere, 288, 1 (1968).

19 S. Blasenbrey and W. Pechhold, Ber. Bunsenges. Phys. Chem. 74, 784 (1970).

20 H. Rehaag and H. A. Stuart, Phys. Z. 38, 1027 (1937).

21 W. Kast and H. A. Stuart, Phys. Z. 40, 714 (1939). 


\section{HETEROGENEITIES IN GLASSY AND MOLTEN POLYMERS}

22 E. Schrader and H. G. Zachmann, Ber. Bunsenges. Phys. Chem. (1975) in press.

23 E. Schrader, Dissertation, Mainz (1970).

${ }^{24}$ E. W. Fischer, J. H. Wentorff, M. Dettenmaier, G. Lieser and I. Voigt-Martin, Polymer Preprint, 15, No. 28 (1975).

25 R. DeSantis and H. G. Zachmann, in preparation.

${ }^{26} \mathrm{~K}$. H. Anthony and E. Kröner in Batelle Conference Report: 'Deformation and fraction in high polymers, (Kronberg 1972). Edited by H. H. Kausch, J. A. Hassel and R. J. Jaffee (1974).

27 P. J. Flory, Proc. Roy. Soc. [London], Ser. A 234, 60 (1956).

28 W. Pechhold and S. Blasenbrey, Kolloid-Z. Z. Polymere, 216-217, 235 (1967).

29 H. Baur, Colloid \& Polymer Sci. 252, 641 (1974).

30 J. Schwarz, Kolloid-Z. Z. Polymere, 251, 215 (1973).

31 Th. G. F. Schoon and E. Kretschmer, Kolloid-Z. Z. Polymere, 197, 43 and 350 (1964); 211, 55 (1965).

32 P. Schmedding and H. G. Zachmann, Kolloid-Z. Z. Polymere, 250, 1105 (1972).

33 H. G. Zachmann, J. Polym. Sci. C: Polymer Symposia No. 43, 111 (1973).

34 P. J. Flory, Statistical Mechanics of Chain Molecules, Interscience: New York (1969).

35 J. Frenkel, J. Chem. Phys. 7, 538 (1939); see also J. I. Frenkel, Kinetische Theorie der Flüssigkeiten, VEB Deutscher Verlag der Wissenschaften: Berlin (1957).

${ }^{36}$ G. S. Y. Yeh and S. L. Lambert, to be published.

37 W. M. Ewers, H. G. Zachmann and A. Peterlin, Kolloid-Z. Z. Polymere, 250, 1187 (1972).

38 W. Frank, H. Gŏddar and H. A. Stuart, Polymer Letters, 5, 711 (1967).

39 W. Frank and H. A. Stuart, Kolloid Z. Z. Polymere, 225, 1 (1968).

${ }^{40}$ R. Hoffmann and W. Knappe, Kolloid-Z. Z. Polymere, 240, 784 (1970).

41 R. Hoffmann, Dissertation, Technische Hochschule Darmstadt (1972).

42 H. Breuer and G. Rehage, Kolloid Z. Z. Polymere, 216-217, 159 (1967).

${ }^{43}$ K.-H. Illers, Makromol. Chem. 127, 1 (1969). 